\title{
La Bibliotheca Alexandrina : du rêve à la réalité
}

\section{Aly Maher El Sayed}

\section{(2) OpenEdition}

\section{Journals}

Édition électronique

URL : https://journals.openedition.org/rbnu/3343

DOI : $10.4000 /$ rbnu.3343

ISSN : 2679-6104

\section{Éditeur}

Bibliothèque nationale et universitaire de Strasbourg

\section{Édition imprimée}

Date de publication : 1 novembre 2010

Pagination : 70-73

ISSN : 2109-2761

\section{Référence électronique}

Aly Maher El Sayed, "La Bibliotheca Alexandrina : du rêve à la réalité », La Revue de la BNU [En ligne], 2 | 2010, mis en ligne le 01 novembre 2010, consulté le 08 août 2021. URL : http:// journals.openedition.org/rbnu/3343; DOI : https://doi.org/10.4000/rbnu.3343

\section{(c) (i) (3)(2)}

La Revue de la BNU est mise à disposition selon les termes de la Licence Creative Commons Attribution - Pas d'Utilisation Commerciale - Partage dans les Mêmes Conditions 4.0 International. 


\section{La Bibliotheca Alexandrina : du rêve à la réalité}

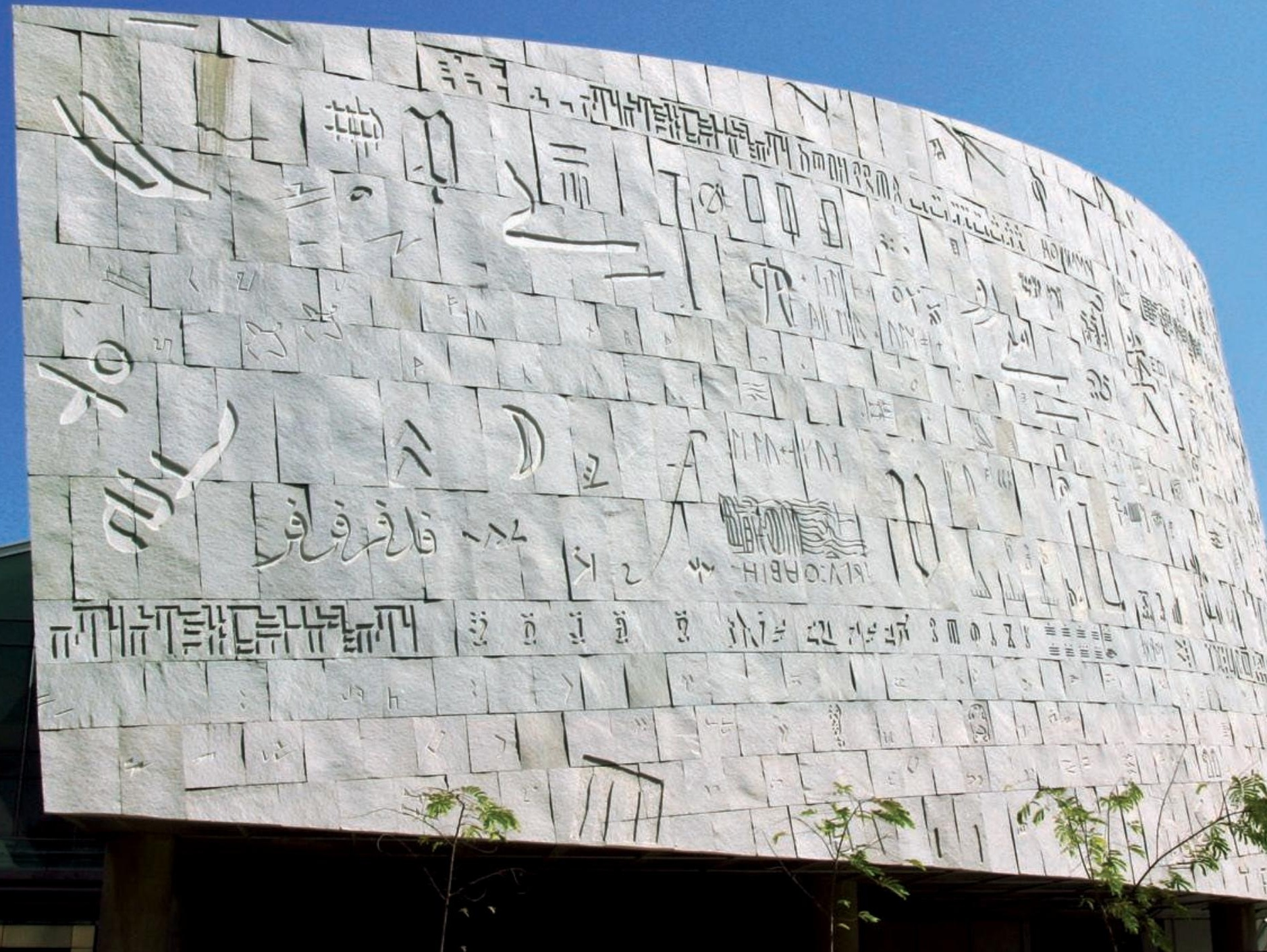




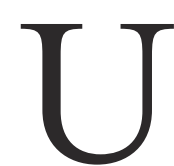

n projet, un rêve fou, démesuré, gigantesque, utopique et extraordinaire, celui de faire revivre la légendaire bibliothèque d'Alexandrie disparue il y a 1600 ans, s'est réalisé grâce à la forte volonté politique égyptienne ainsi qu'à l'appui de la communauté internationale.

L'ancienne bibliothèque avait incarné pendant plus de six siècles l'apogée du savoir et avait été le symbole de l'universalisme et surtout du triomphe de l'esprit et de l'intelligence. Comme un phénix, la nouvelle bibliothèque renaît des cendres de son ancêtre. Elle ouvre ses portes le 16 octobre 2002 en présence d'un grand nombre de chefs d'Etat, du directeur général de l'UNESCO et d'éminents intellectuels du monde entier, qui tous sont venus célébrer avec l'Egypte la concrétisation du rêve qui a, pendant longtemps, hanté l'esprit des hommes. Avec son architecture futuriste appréciée dans le monde entier, cette nouvelle bibliothèque se veut l'héritière des traditions de sa prestigieuse devancière. Elle se veut un lieu de dialogue, de liberté d'expression, d'aventure intellectuelle, d'art et de sciences, et œuvre à réunir en un seul lieu tout le savoir du monde, tout en devenant la fenêtre du monde sur l'Egypte et la fenêtre de l'Egypte sur le monde.

Pour ce faire, ce complexe scientifique et culturel est beaucoup plus qu'une simple bibliothèque : dans un établissement pouvant contenir plusieurs millions de livres, il abrite un centre d'archivage de l'internet, six bibliothèques spécialisées (pour les arts, le multimédia et l'audiovisuel, les microformes, les livres rares et les collections spéciales, mais aussi pour le public des jeunes et des malvoyants), quatre musées (consacrés aux antiquités, aux manuscrits, à l'histoire des sciences et à l'ancien président Sadate), un planétarium, des espace appelés ALEXploratorium (pour que les enfants découvrent la science et se familiarisent avec elle), Culturama (premier panorama culturel composé de neuf écrans à multicouches numérisées interactives), VISTA (immersion virtuelle des applications des sciences et de la technologie qui crée un environnement interactif de la réalité virtuelle), ceci sans compter quinze expositions permanentes, quatre galeries d'expositions temporaires, un centre de conférences pour des milliers de personnes, huit centres de recherche académique, un Forum du dialogue...
De plus, la nouvelle bibliothèque d'Alexandrie héberge un certain nombre d'institutions telles que l'Academia Bibliothecae Alexandrinae (ABA), la Société arabe pour l'éthique des sciences et de la technologie (ASEST), la Fondation euroméditerranéenne pour le dialogue entre les cultures Anna Lindh (ALF), l'Institut des études pour la paix, affilié au Mouvement Suzanne Mubarak des femmes pour la paix, le Projet de recherches médicales HCM, le Centre René-Jean Dupuy pour le droit et le développement, le Bureau régional arabe de l'Académie des sciences pour les pays en développement (TWAS-ARO), le Bureau régional de la Fédération internationale des associations de bibliothécaires et des bibliothèques (FIAB/IFLA), le Secrétariat des Commissions nationales arabes pour l'UNESCO, le Réseau du Moyen-Orient et de l'Afrique du Nord pour l'économie environnementale (MENANEE).

Le travail accompli depuis l'inauguration et durant les huit dernières années a répondu à ces ambitions. Avec plus de 700 manifestations annuelles dans les domaines des sciences, de l'art, de l'économie, de la politique et des réformes en général, la BA est effectivement devenue un espace de dialogue et de rencontre entre les peuples et les cultures. Elle est aujourd'hui la plaque tournante qui a restauréle lustre cosmopolite de la ville d'Alexandrie. Plus qu'une bibliothèque, la $\mathrm{BA}$ du XXI ${ }^{e}$ siècle constitue un centre d'excellence culturelle vivant et multidisciplinaire, ouvert sur la société égyptienne, sur le grand large méditerranéen et sur la communauté internationale. Ainsi, l'institution naissante a réussi en peu de temps à tisser un véritable réseau de coopération et de partenariats nationaux et internationaux avec d'illustres institutions culturelles et scientifiques à travers le monde.

La variété des activités de la BA souligne, entre autres, le caractère multidisciplinaire de cette institution sui generis.

Afin de réaliser ses objectifs et pour demeurer à l'avant-garde des avancées techniques, la BA a mis l'accent dès le premier jour sur la nécessité de relever les défis de l'ère numérique, en mettant en place des bases de données électroniques ; étant en quelque sorte " née numérique ", la bibliothèque d'Alexandrie a beaucoup œuvré pour asseoir sa place dans le monde désormais incontournable de 


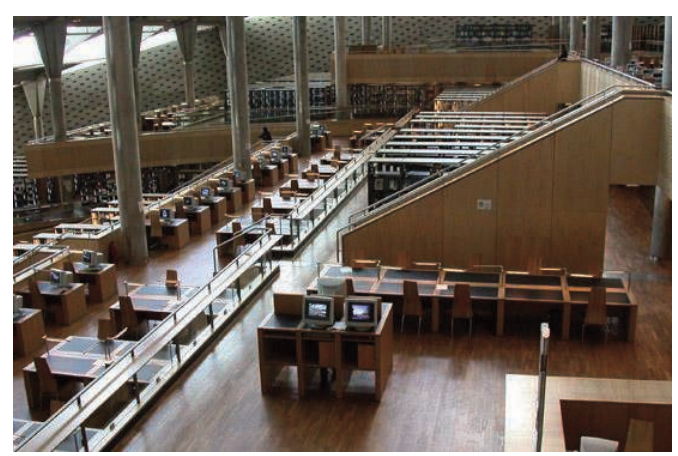

Vue générale de la salle de lecture

l'informatique, en numérisant une part importante de son fonds.

Par ailleurs, elle vise à développer ses collections en tenant compte de son appartenance égyptienne, euro-méditerranéenne, arabe et africaine.

Car si la BA a des dimensions universelles importantes, elle reste toutefois ancrée dans son environnement égyptien. La présence et l'action de cette prestigieuse institution, en ce sens, a modifié le paysage alexandrin et a créé un véritable pôle d'attraction autant pour les Egyptiens d'Alexandrie et d'ailleurs que pour les milliers de touristes arabes et étrangers qui ont retrouvé le chemin de la ville fondée par Alexandre le Grand. Les salles de lecture, les expositions, les colloques, les conférences, les manifestations culturelles attirent plus d'un million de visiteurs par an dont une grande majorité de jeunes.

Il faut par ailleurs noter que la dimension égyptienne de la BA est soulignée par la présence d'un musée consacré au président Sadate, par la mise en œuvre d'un projet audiovisuel électronique sur la mémoire de l'Egypte et son histoire moderne, par des publications et des sites électroniques sur les personnalités marquantes de l'histoire de l'Égypte dans les domaines politique, économique, intellectuel, médiatique et artistique.

Aujourd'hui, huit ans après son inauguration, la nouvelle bibliothèque jouit d'une réputation mondiale comme étant un centre d'excellence. Afin de préserver ce label, il faut un travail dur et assidu fondé sur les réalisations déjà achevées. Il faut en particulier approfondir davantage la coopération avec les instances internationales et les institutions culturelles d'Europe, d'Amérique, d'Asie et d'Afrique.
A cet égard, la coopération exceptionnelle qui existe entre la BA et la Bibliothèque nationale de France est un modèle du genre. En effet, la BA a bénéficié en 2010 du don somptueux et extraordinaire de 500000 ouvrages offerts par la BnF. C'est le plus grand don de livres de l'histoire, un don qui dépasse de loin en nombre les 200 ooo parchemins offerts à Cléopâtre par Marc Antoine. Plus qu'un acte culturel, ce don incarne un geste politique que les Egyptiens apprécient à sa juste valeur. Pour le valoriser, et dans le cadre de la convention-cadre de coopération entre les deux institutions, un plan de formation, des stages professionnels, un encadrement du catalogage de ces livres offerts ont été mis en place. Ce don exceptionnel a fait de la BA la quatrième bibliothèque francophone du monde, hors de France.

La France a été ainsi présente dès le début du projet, et elle le reste aujourd'hui : à Assouan en 1990, le président Mitterrand a exprimé l'appui de son pays pour réaliser ce rêve, au cours de la réunion internationale qui a donné le coup d'envoi à la construction de la Bibliotheca Alexandrina ; par la suite, lors des différentes phases de construction et de l'établissement d'un système électronique, la France était partie prenante ; le 16 octobre 2002, le président Mubarak, accompagné par le président Chirac et d'autres chefs d'Etat et de gouvernement, des intellectuels éminents et des artistes mondialement reconnus, a inauguré la Bibliotheca Alexandrina. Enfin, en 2010, le don prestigieux de la BnF vient assurer, une fois de plus, la continuité du soutien français à la BA.

Le rêve continue..., bien qu'il soit aujourd'hui réalité, mais une réalité aussi belle qu'un rêve...

\section{Aly Maher El Sayed}




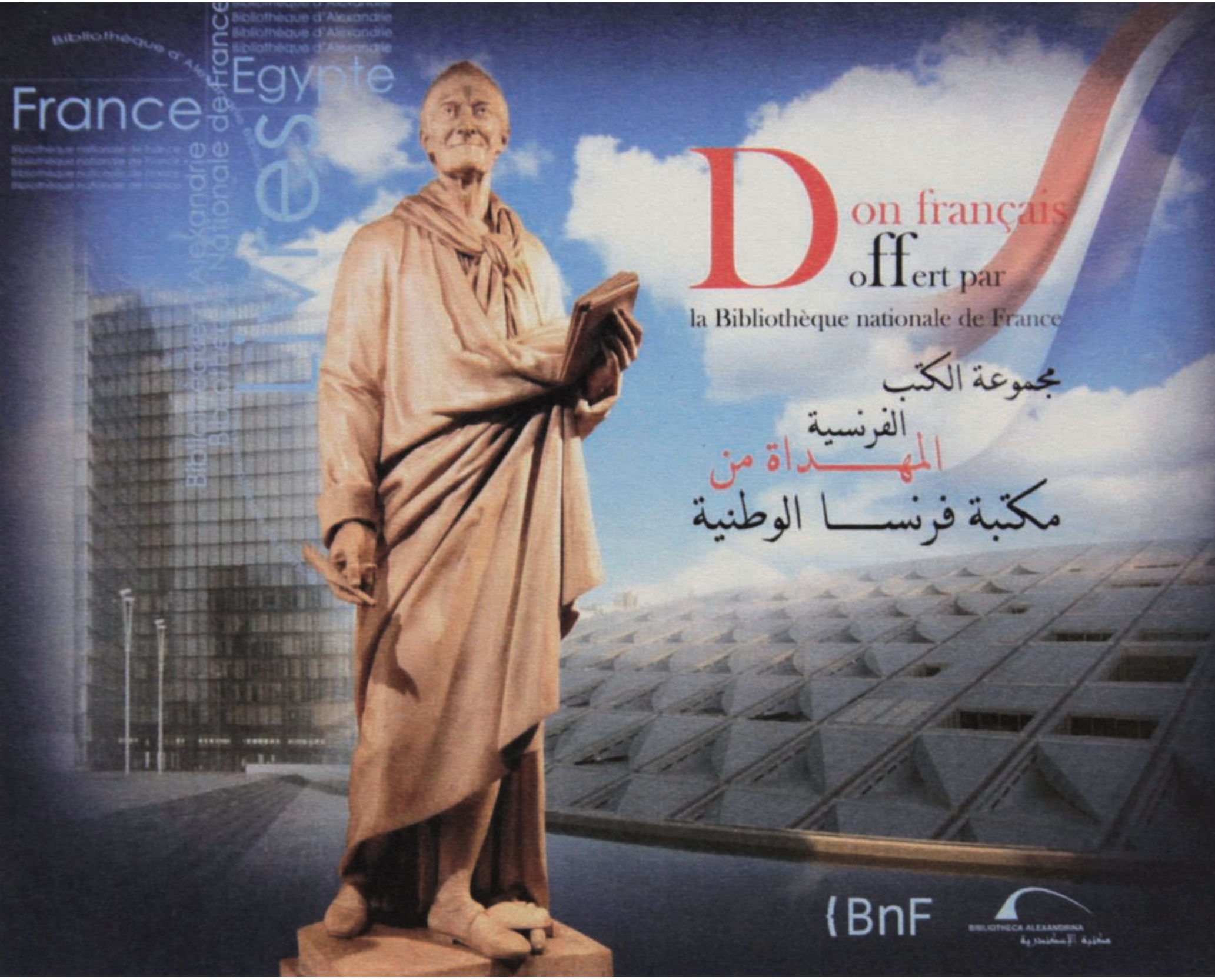

